\title{
Linx
}

Revue des linguistes de l'université Paris X Nanterre

$12 \mid 2002$

"Comme la lettre dit la vie »

\section{Je dis que ... Je pense que ... Le je narrateur, auteur, témoin et personnage des chroniques}

\section{Sophie Marnette}

\section{OpenEdition}

\section{Journals}

Édition électronique

URL : http://journals.openedition.org/linx/1302

DOI : $10.4000 /$ linx.1302

ISSN : 2118-9692

\section{Éditeur}

Presses universitaires de Paris Nanterre

\section{Édition imprimée}

Date de publication : 1 octobre 2002

Pagination : 186-194

ISSN : 0246-8743

\section{Référence électronique}

Sophie Marnette, « Je dis que ... Je pense que ... Le je narrateur, auteur, témoin et personnage des

chroniques », Linx [En ligne], 12 | 2002, mis en ligne le 10 octobre 2012, consulté le 19 avril 2019. URL : http://journals.openedition.org/linx/1302 ; DOI : 10.4000/linx.1302

Ce document a été généré automatiquement le 19 avril 2019.

Département de Sciences du langage, Université Paris Ouest 


\title{
Je dis que ... Je pense que ... Le je narrateur, auteur, témoin et personnage des chroniques
}

\author{
Sophie Marnette
}

\section{Introduction}

1 Contrairement à bon nombre de textes médiévaux restés anonymes, les chroniques des 13 $\mathrm{e}, 14^{\mathrm{e}}$ et $15^{\mathrm{e}}$ siècles présentent généralement le nom de leur auteur. Il s'agit de noms référant à des personnes historiques plus ou moins notables et dont les motifs moraux ou politiques sont plus ou moins familiers de leurs contemporains et de nous, lecteurs modernes. Villehardouin, Joinville, Froissart et Commynes sont ainsi bien mieux connus que des chroniqueurs comme Robert de Clari, Enguerrand de Monstrelet ou le Héraut de Berry mais tous ont pris soin de souligner leur identité dans leur prologue ou leur épilogue. La présence de noms d'auteur dans les chroniques semblerait dès lors éviter tout conflit entre notre notion moderne d'auteur en tant qu'origine unique et créatrice du texte, et la notion ambiguë d'auteur que construisent des textes médiévaux sans attribution ou d'attribution multiple (e.g. Chrétien de Troyes et Geoffroy de Leigni pour Le Chevalier à la charrette) ${ }^{1}$.

2 Ceci n'empêche bien sûr aucunement les remaniements, qu'ils soient opérés par les chroniqueurs eux-mêmes, au cours de versions successives, ou bien par les scribes copistes. De fait, alors qu'un je narrateur unique est directement mis en scène par le texte, la figure de l'auteur et ses motifs ne peuvent être reconstruits qu'ultérieurement avec plus ou moins de bonheur sans pouvoir toujours distinguer entre les divers remaniements, ni les possibles ajouts de copistes. La distinction entre narrateur et auteur est particulièrement nette dans les deux premières chroniques de la Prise de Constantinople (Clari et Villehardouin) où les références à l'auteur se font à la $3^{\mathrm{e}}$ pers. tandis que la voix narrative est à la $1^{\text {ère }}$ pers. du sg. (je) et plus souvent encore à la $1^{\text {ère }}$ pers. du pl. (nous emphatique). Comme l'a notamment fait remarquer Michèle Perret (1981: 3) c'est avec 
Joinville que s'effectue dans le genre des chroniques la coïncidence entre le nom de l'auteur et le je narrateur.

3 Cependant, le je narrateur n'est pas seulement celui qui raconte l'histoire en tant que pur locuteur mais c'est aussi un être du monde qui fait parfois part de son avis sur son récit et le lie au monde extérieur. Ces opinions peuvent être relativement impersonnelles mais elles sont parfois aussi annoncées par des marqueurs modaux et évidentiels forts, tels que je dis que, je crois que, etc. En ce sens les chroniques, même les plus délibérément objectives, sont aussi des jugements sur l'Histoire.

4 Enfin, c'est aussi à partir de Joinville que le je mis en scène par le texte devient à la fois celui qui raconte l'histoire (narrating self) et celui qui l'a vécue en tant que témoin ou même en tant que participant (experiencing self). Ces deux derniers rôles étaient également exprimés à la $3^{\mathrm{e}}$ pers. dans les chroniques de Clari et de Villehardouin. Tous les chroniqueurs n'ont pas assisté aux événements qu'ils racontent et même chez Joinville, certaines anecdotes relèvent de ce qu'il a entendu dire ou de ce qu'il a lu. Toutefois tous les chroniqueurs insistent à un moment ou à un autre sur le fait qu'ils étaient présents à certains événements ou qu'ils ont interrogé des témoins dignes de foi. Nous verrons que c'est ce lien de plus en plus fort entre je narrateur, je auteur et je témoin ou participant qui garantit l'historicité de ces récits.

Le présent article étudie neuf extraits de chroniques françaises allant du $13^{\mathrm{e}}$ au $15^{\mathrm{e}}$ siècles et y examine les différents rôles joués par un je 'caméléon' qui peut-être narrateur, auteur, témoin ou même personnage du récit. ${ }^{2}$ Cette étude se situe bien entendu dans la lignée des beaux travaux de Michèle Perret sur la position du narrateur dans des textes médiévaux comme Le Bel Inconnu (Perret 1996) et La Vie de Saint Louis (Perret 1981) et sur l'énonciation en français médiéval (Perret 1988, 1993, 1995, 1997).

\section{Chroniques du $13^{e}$ siècle}

6 Pour comprendre l'évolution des chroniques, il faut remonter au $13^{\mathrm{e}}$ siècle qui vit la naissance des romans en prose et des chroniques en langue vernaculaire sous l'équation commune 'prose=vérité'. En effet, comme comme l'a bien décrit Spiegel (1993), la prose vernaculaire du $13^{\mathrm{e}}$ siècle apparaît dans le sillage de la prose latine historique et/ou religieuse: traduction de la Bible, vies de saint, historiographie ${ }^{3}$. Dans des travaux précédents (Marnette 1998, 1999), j'ai analysé les caractéristiques linguistiques et narratologiques des premières chroniques en prose en me penchant sur la position du narrateur ainsi que sur les points de vue selon lesquels le contenu du récit est filtré (focalisation). En voici un bref résumé.

7 Les chroniques de Constantinople de Clari et de Villehardouin, qui sont les plus anciennes, emploient un nous exclusif et autoritaire pour référer au narrateur de préférence à je, et elles utilisent la $3^{\mathrm{e}}$ pers. sg. pour désigner l'auteur-témoin qui est aussi un personnage de l'histoire racontée $e^{4}$.

Villehardouin, § 120, lignes 1-3.

Et bien testimoigne Joffrois li mareschaus de Champaigne qui ceste oevre dita, que ainc n'i menti de mot a son escient, si com cil qui a toz les conseils fu, (...). 
Clari, § 120, lignes 4-10.

(...) chis qui i fu et qui le vit et qui l'oï le tesmongne, Robers de Clari, li chevaliers, et a fait metre en escrit le verité, si comme ele fu conquise; et ja soit chou que il ne l'ait si belement contee le conqueste, comme maint boin diteeur l'eussent contee, si en a il toutes eures le droite verité contee, et assés de vérités en a teutes qu'il ne peut mie toutes ramembrer.

Ces textes illustrent donc bien les débuts de l'emploi de la prose en tant que modalité discursive porteuse d'une vérité impersonnelle, unique et immanente. Ce qui ne les empêche pas, par ailleurs, d'insérer subtilement une série d'interprétations subjectives qui visent à faire passer un message politique bien précis auprès des auditeurs-lecteurs. ${ }^{5}$.

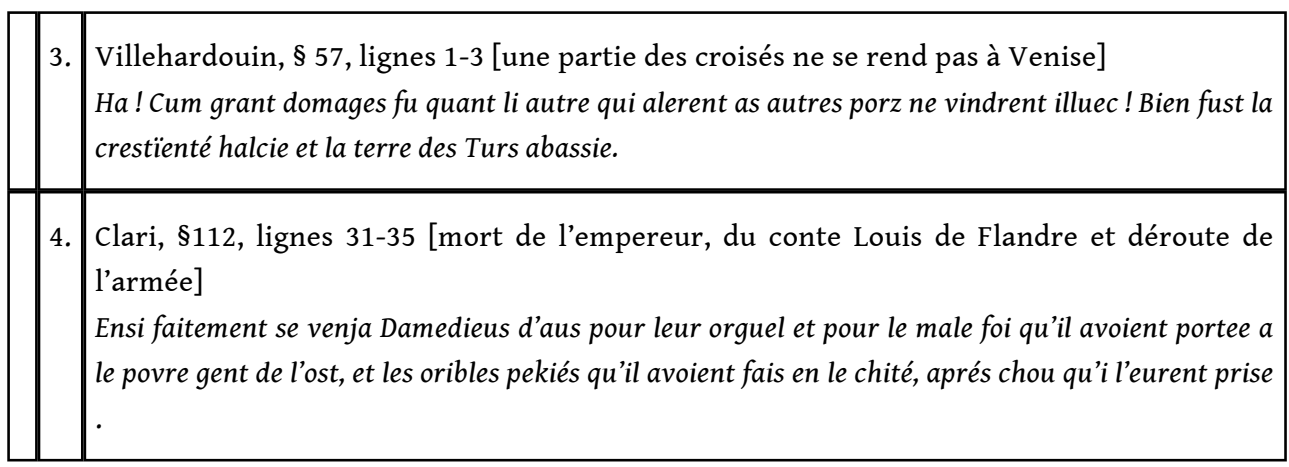

9 Les chroniques de Clari et Villehardouin instaurent donc l'instance narrative en tant que voix neutre et indivisible, qui se base sur une source autoritaire (témoin oculaire), permettant dès lors l'équation entre la notion de prose et les notions d'autorité, de réalité et de vérité uniques.

\section{Les Chroniques des $14^{\mathrm{e}}$ et $15^{\mathrm{e}}$ siècles}

Les chroniques des $14^{\mathrm{e}}$ et $15^{\mathrm{e}}$ siècles mettent en scène un je narrateur qui est à la fois l'auteur qui dicte ou met par écrit le récit, un moraliste dans le présent, le témoin direct ou indirect des événements racontés ainsi qu'un personnage qui a quelquefois participé aux actions du passé. Ces chroniques conservent bien tous les traits dominants propres au genre : visée politique, événements vus de l'extérieur, emploi du passé comme temps non marqué du récit et donc, séparation nette entre le temps de la narration et le temps du récit. Toutefois, elles sont écrites à un moment où la prose n'est plus nécessairement vue comme un facteur de vérité en tant que telle, ni d'ailleurs comme excluant le je. Dans les chroniques de Joinville, Froissart et Commynes, la fréquence des $1^{\text {ères }}$ pers. du sg. référant au je narrateur-auteur-moraliste est toujours supérieure ou égale à $2 \%$, un chiffre qui augmente lorsqu'on compte aussi les références au chroniqueur en tant que personnage participant aux événements du passé ou encore en tant que témoin direct et indirect ${ }^{6}$. Dans ces textes, la fréquence de la $1^{\text {ère }}$ pers. du sg. est toujours plus élevée que celle de la $2^{\mathrm{e}}$ pers. de sorte que c'est le je narrateur, témoin et personnage qui est plus important que les auditeurs-lecteurs ${ }^{7}$. Même dans les chroniques de Monstrelet et du Héraut de Berry, où la fréquence de la $1^{\text {ère }}$ pers. du sg. est très peu élevée en dehors des prologues, le je n'apparaît pas uniquement en tant que narrateur mais aussi en tant qu'auteur (nom propre) et témoin (voir ci-dessous) ${ }^{8}$. Ce qui est souligné dès lors, c'est la position 
essentielle du je qui assiste et/ou participe à l'histoire, qui la met par écrit et donc l'objectivise, et enfin qui la juge et la rattache à son époque par des commentaires moraux.

\section{Le je narrateur 'pur'}

11 La fonction purement narrative peut s'exprimer à la $1^{\text {ère }}$ pers. du sg. ou à la $1^{\text {ère }}$ pers. du pl. et elle transparaît également dans les adresses aux auditeurs-lecteurs.

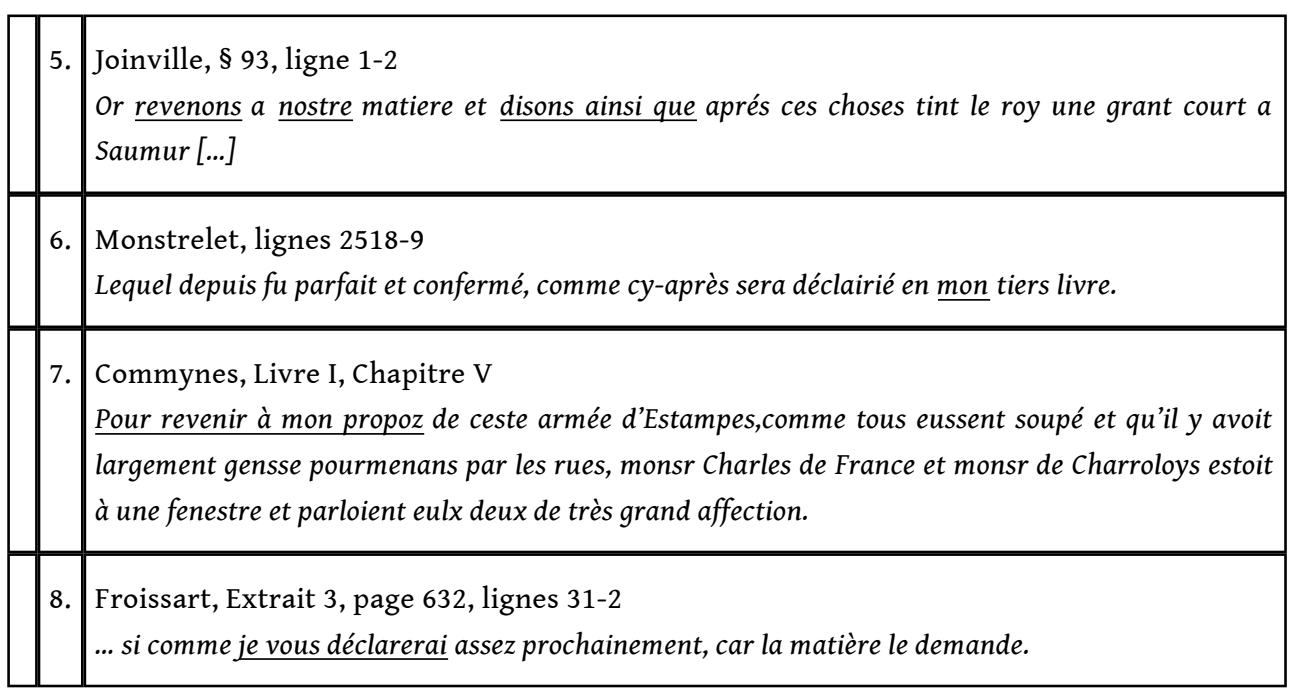

12 Cette fonction est généralement importante ( $2 \%$ des $1^{\text {ères }}$ pers. ou plus dans Commynes, Froissart 2 et 3, Clari, Joinville). Elle est majoritaire dans les chroniques de Constantinople et correspond à un quart ou un tiers de l'ensemble des $1^{\text {ères }}$ pers. sg. dans Froissart 1, Froissart 3, Monstrelet et Commynes. Seuls les textes de Joinville et Froissart 2 (Bearn) y consacrent moins de $10 \%$ des occurrences des $1^{\text {ères }}$ pers. sg. car ces textes réfèrent majoritairement au chroniqueur en tant que témoin ou participant aux évévements ( experiencing self).

\section{Le je narrateur-auteur}

13 Comme je l'ai déjà souligné, ce qui distingue les chroniques des $14^{\mathrm{e}}$ et $15^{\mathrm{e}} \mathrm{s}$. des chroniques de Clari et Villehardouin, c'est la coïncidence entre le je représentant le narrateur avec la référence au nom et à la fonction d'auteur 9 .

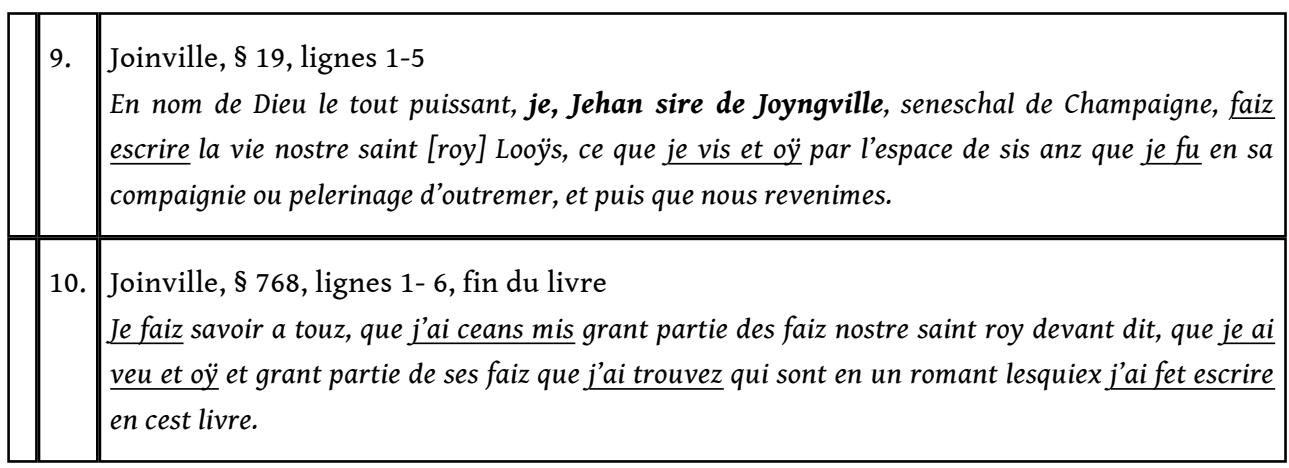


11. Froissart, Livre $1, \S 311$

Pluiseur gongleour et enchanteour en place ont chanté et rimet lez guerres de Bretaigne et coromput par leurs chançons et rimes controuvees, le juste et vraie histoire, dont trop en desplaist à monseigneur Jehan le Biel qui le coummencha a mettre en prose et en cronique et à moy, sire Jehan Froissart, qui loyaument et justement l'ay poursuiwi à mon pooir. Car leur rimmez et leurs canchons controuvees n'ataindent en riens le vraie matere més velle ci si comme nous l'avons faite et achievee par le grande dilligensce que nous y avons rendut car on n'a riens sans fret et sans pene. Jou, sire Jehans Froissars, darrains venus depuis monseigneur Jehan le Bel en cel ouvraige, ai ge allé et cherchiet le plus grant partie de Bretaingne et enquis et demandé as seigneurs et as hiraux les gerrez, les prises, les assaux, les envaies, les bataillez, les rescousses et tous les biaux fés d'armes qui sont avenut mouvant sus l'an de grasce mil.CCC.XL poursieuwans jusquez à le darrainne datte de ce livre, tant à le requeste de mes dis seigneurs et à ses fraix que pour me plaisance acomplir et moy fonder sus title de verité et dont j'ay estet grandement recompenssés.

12. Monstrelet, Prologue du second livre

[...] Pour lesquelles ramener à mémoire et recordacion véritable, je Enguerran de Monstrelet, faisant ma résidence en la cité de Cambray, qui autreffois ay prins laborieux plaisir à faire mettre par escript, par manière de cronique, les mervilleuses adventures et vaillances d'armes dignes de louenges et recordacion, advenues au très crestien royaulme de France, ès pays voisin, et ès marche loingtaines, tant de la crestienté comme d'aultre loy, à mon petit entendement, sans polir les choses ne yssir hors de la matière, mais mettant le fait directement en ensuyvant les récitacions qui faictes en ont esté à moy par plusieurs hommes nobles et autres notables personnes et aussy par roys-d'armes, héraulx et pousuyvans dignes de foy et de crédence, qui ont esté presens aux besognes, me suis remis à continuer et à poursuyvir ce que de long temps avoie et ay encommencée, et a entendre les besongnes pour compiler ces présentes hystoires; qui se comprennent comme on pourra veoir à les lyre et oyr, en batailles mortelles, désolacions de plusieurs églises, cités, villes et forteresses, dépopulacion de moult de pays et aultres merveilles piteuses à recorder, [...] . Et pour ces raisons, ay voulu mettre et par exposer mon temps, comme dit est, en persévérant en ycelle occupacion. Car, avec ce, ay assez apperceu et veu par expérience, ce que aucuns princes et seigneurs de grande auctorité et de divers estas ont prins plaisir à en veoir et oyr aulcune chose, jà soit-il que ce ne soit pas sans paine de enquérir, veiller et travailler, que telz fais se peussent ainsy par ordre assembler. Toutefois, de tant [moins] griève le travail comme l'acteur y prend, quand il le fait libéralement en y prenant plaisir

Si commencera ycellui mon second livre, ou mois d'octobre mil quatre cens et vint et deus [...]. 


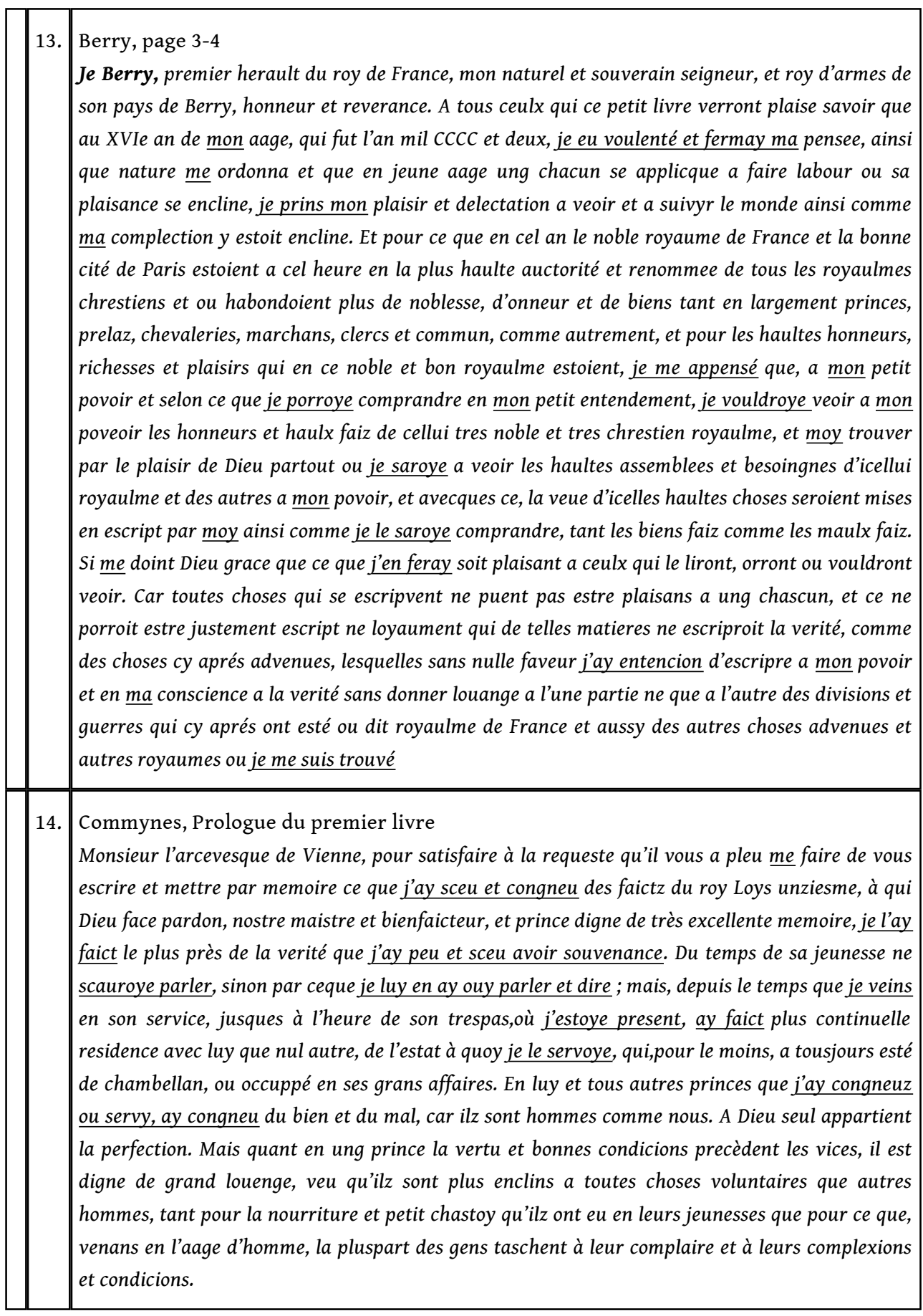

C'est parce qu'il a assisté en personne à certains événements (témoignage direct) ou qu'il les a entendus raconter par des témoins (témoignage indirect) ou encore parce qu'il se base sur certains écrits (e.g. un 'roman' pour Joinville [10], la chronique de Jean le Bel pour Froissart 1 [11]) que le je narrateur-auteur est le garant de la vérité de son récit (voir aussi Blanchard 1991 : 197). Un fait qui est renforcé par l'emploi de la formule juridique du type 'je + nom propre' [9,10,11,12,13], comme le remarque fort à propos MarchelloNizia (1984: 19). Il faut souligner que les formules performatives des chroniques sont toujours suivies par une expression à la $1^{\text {ère }}$ pers. qui insiste sur la fonction de témoin direct ou indirect de l'auteur ${ }^{10}$. Ainsi, la chronique de Monstrelet contient-elle très peu de 
$1^{\text {ères }}$ pers. du sg. et de $2^{\text {es }}$ pers. $(0.7 \%$ et $0.3 \%)$ mais le texte débute bien par la formule juridique, je Enguerran de Monstrelet [12], et si trois quart des $1^{\text {ères }}$ pers. du sg. de l'extrait réfèrent au je narrateur, un quart désigne le je témoin, contrairement aux anciennes chroniques de Clari et Villehardouin qui emploient la $3^{\mathrm{e}}$ pers. sg. dans ce dernier cas (voir $[1,2])$. De même, si la majorité des $1^{\text {ères }}$ pers. du sg. qui apparaissent dans le texte du Héraut de Berry sont restreintes au prologue, elles sont clairement associées à une formule performative ainsi qu'à la position d'auteur (64\%) et de témoin (12\%) [13].

Cependant le je auteur n'apparait pas seulement dans le texte par le biais d'un nom propre. Il est aussi utilisé pour référer au narrateur-auteur en tant que moraliste qui a une opinion à propos des événements racontés (voir section suivante), en tant qu'être du monde qui poursuit un but précis en racontant certains événements, qui s'adresse à un patron particulier ([14]) ou encore qui décrit la mise en œuvre de son texte. Ainsi, dans son prologue le chroniqueur expose-t-il souvent pourquoi il a décidé, en tant qu'individu, de composer son œuvre, référant à une inclination ou une aptitude particulière ou encore à sa position idéale auprès de personnages historiques précis (Louis IX pour Joinville, Louis XI pour Commynes, la reine d'Angleterre pour Froissart) et à ses qualités de voyageur (Froissart, Monstrelet, Berry). Allant au-delà des simples préoccupations de narrateur (cohésion du texte: ce que je vais vous conter ou ce que je vous ai conté), le je narrateur-auteur se préoccupe donc aussi de la cohérence globale de son œuvre, un souci qui n'était pas présent dans les textes de Villehardouin et Clari.

\section{Le je moraliste}

Comme on l'a vu dans les exemples [3,4] des chroniques de Constantinople, même les chroniques en apparence les plus objectives peuvent exprimer les opinions du chroniqueur. Les chroniques des $14^{\mathrm{e}}$ et $15^{\mathrm{e}}$ sont parfois plus explicites et ne se contentent plus de formules exclamatives mais utilisent des formules réflexives qui mettent en scène les opinions de l'auteur via la voix du narrateur.

\begin{tabular}{|l|l|ll|}
\hline 15. & $\begin{array}{l}\text { Joinville, § 18, lignes 1-3 } \\
\text { Or diz je a vous mon seigneur le roy de Navarre, que je promis a ma dame la royne vostre mere a } \\
\text { cui Diex bone merci face que je feroie cest livre. }\end{array}$ \\
\hline 16. & $\begin{array}{l}\text { Froissart, Extrait } 2: \text { Bearn, page 534, ligne } 7 \\
\text { Le comte Gaston de Foix, dont je parle, en ce temps que je fus devers lui, avoit environ cinquante- } \\
\text { neuf ans d'âge. Et vous dis que j'ai en mon temps vu moult de chevaliers, rois, princes et autres; } \\
\text { mais je n'en vis oncques nul qui fût de si beaux membres, de sui belle forme ni de si belle taille et } \\
\text { viaire, bel, sanguin et riant, ... }\end{array}$ \\
\hline 17. & $\begin{array}{l}\text { Froissart, Extrait 3, pages } 609, \text { lignes 8-9 } \\
\text { Et je vous dis que la litière de la roine étoit très riche et bien aournée et toute descouverte. }\end{array}$ \\
\hline 18. & $\begin{array}{l}\text { Joinville, § 278, ligne } 6 \\
\text { Et ces choses vous ai-je ramentu pource que je croi que Dieu li otroia, si com vous pouez avoir veu } \\
\text { ci-devant. }\end{array}$ \\
\hline
\end{tabular}




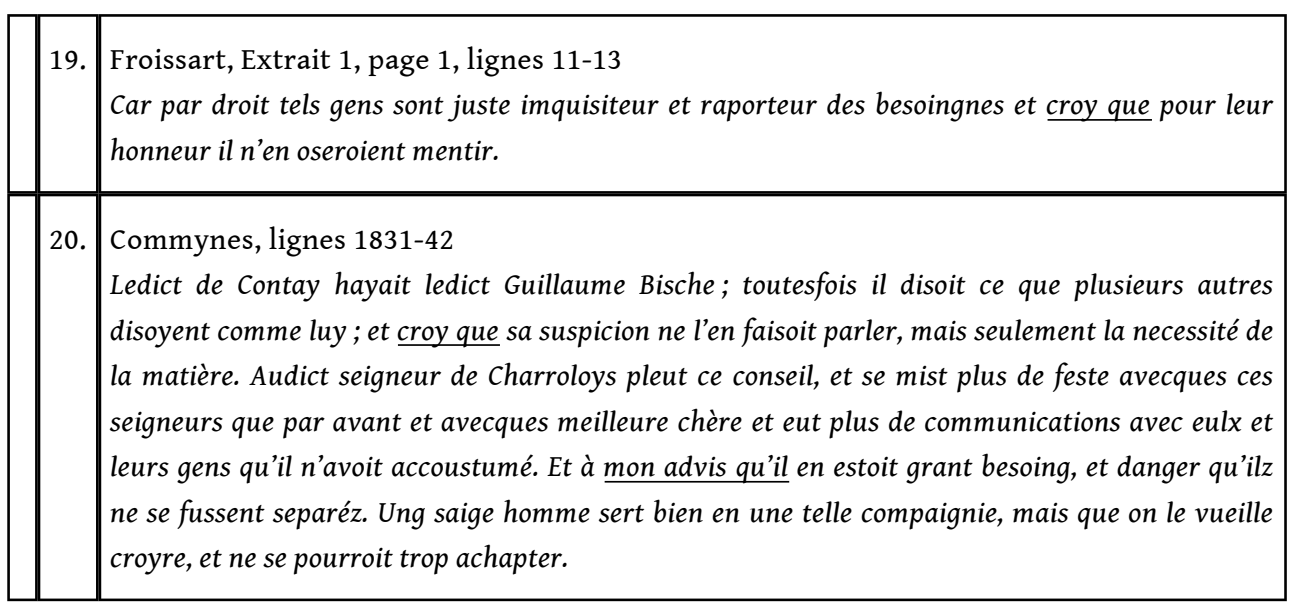

17 Ainsi dans un énoncé comme Je vous dis que $X$, le pronom je désigne-t-il à la fois le narrateur en tant que tel (celui qui raconte, qui 'dit') mais aussi le narrateur en tant qu'être du monde qui est l'énonciateur d'un certain discours ou qui a certaines opinions $[15,16,17]^{11}$. Ce qui permet donc au je d'exhiber son propre discours face aux discours d'autrui et ce par le biais d'un acte de langage dans le présent de l'énonciation. On trouve aussi des expressions comme je crois (que), il m'est avis (que) $[18,19,20]$. Le point commun de ces deux types d'expressions est qu'elles contiennent un verbe au présent et à la 1ère pers. du sg. et qu'il s'agit donc de ce qu'on pourrait appeler des discours rapportés « réflexifs » qui impliquent une théâtralisation des paroles et des pensées du locuteur ${ }^{12}$.

Les expressions du type je dis que se répartissent de la manière suivante : Joinville $0.2 \%$, Froissart $20.5 \%$, Froissart $30.2 \%$, Commynes $0.4 \%$; les expressions du types je crois que, il m'est avis que, il me semble que se répartissent comme suit: Clari $0.1 \%$, Joinville $0.1 \%$, Froissart $10.4 \%$, Monstrelet $0.1 \%$, Commynes $1.4 \%$ : si les fréquences de ces expressions sont en fait peu élevées dans les chroniques, elles sont néanmoins nettement plus nombreuses que dans les textes de fiction qui font partie de mon corpus élargi (Marnette 2002). Notons la haute proportion de ces expressions réflexives dans le texte de Commynes (1.4\%), ce qui montre qu'il s'agit bien d'une réflexion personnelle sur l'histoire racontée, c'est-à-dire d'une œuvre de mémorialiste.

\section{Le je témoin et participant au récit}

Le je narrateur peut à certains moment avouer son ignorance de certains faits parce qu'il n'était pas présent aux événements racontés ou bien parce qu'il ne se souvient pas de tout $[21,22]$. La sincérité (toute apparente) de ces remarques renforce d'une certaine manière la position du je narrateur en tant que garant du récit : il ne peut humainement tout se rappeler et ne peut jurer que de ce qu'il a vu de ses yeux ou entendu dire par des témoins dignes de confiance. Il n'empêche qu'une grande quantité de ce qui est raconté relève d'un choix subjectif du narrateur-auteur qui sélectionne les informations et parfois s'en justifie [22].

21. Commynes, Livre I, Chapitre XV

Je ne sçay de ces choses que ce qu'il m'en a compté, car je n'estoie point sur les lieux. 
22. Froissart, Extrait $1, \S 1$, lignes $47-9$

(...) et pluissieurs hauls barons et bacelers, desquelz je ne puis mie ossi de tous parler car trop porois ma principaul matere ensonniier.

L'impossibilité de rapporter telles quelles les paroles entendues explique sans doute que le narrateur des chroniques ne prétende pas citer fidèlement les discours des personnages par le biais du discours direct, mais préfère les inclure dans sa narration grâce au discours indirect, en employant ses propres mots et en se réservant de cette manière le contrôle du discours ${ }^{13}$. Il s'agit d'un problème dont est conscient un chroniqueur comme Froissart puisqu'il décrit minutieusement dans le Voyage de Béarn la manière dont il prend note, chaque soir, des récits racontés durant la journée par les personnes avec lesquelles il voyage (Froissart 2, p. 525, voir ci-dessous). On peut certainement ajouter à cela le fait que certains chroniqueurs citent des discours qui leur ont été rapportés par des témoins intermédiaires, ce qui rend évidemment impossible le retour au discours supposément originel. De plus, le discours originel a pu être prononcé dans des langues (ou dialectes) autres que le français, un problème qui s'efface aisément au sein du discours indirect ${ }^{14}$. Dans le même ordre d'idées, les pensées des personnages sont peu souvent représentées dans les chroniques puisque cela demanderait un degré certain d'invention de la part de l'auteur. Enfin, on peut aussi noter que l'emploi élevé du discours direct par Monstrelet n'est pas une exception à ces remarques car il s'agit de discours écrit: des lettres officielles et autres documents juridiques qui ont pu être conservés ailleurs sur un support écrit et qui n'entraînent donc pas d'effort de mémoire. Bref, ce qui rend les discours vrais, ce n'est pas qu'ils sont cités tels quels (ce qui est impossible) mais qu'ils sont rapportés par le je narrateur en tant que témoin direct ou indirect.

21 Comme je l'ai déjà indiqué, dans nos textes, la fonction de témoin est indissociable de celle d'auteur. On trouve cependant plusieurs références au rôle de témoin en dehors du simple nom d'auteur cité dans le prologue : Joinville (1.3\%), Froissart $3(0.9 \%)$, Monstrelet (0.2\%), Berry (0.2\%), Commynes (3.6\%). Il s'agit d'une catégorie difficile à évaluer car le je peut-être un témoin direct, auquel cas il est parfois confondu avec le rôle de participant. De fait, lorsque Joinville, Froissart et Commynes racontent qu'ils ont pris part à certains événements, c'est forcément pour renforcer leur position de témoin de l'Histoire et donc leur crédibilité. Joinville n'est cependant pas loin de raconter sa propre histoire à certaines reprises, davantage que celle de Saint Louis (Perret 1981:18, 26). D'autres exemples sont plus clairs quant à la position du chroniqueur en tant que témoin «externe»[23]. Dans tous les cas, ces exemples de témoignages directs et de participation aux événements racontés nous donne une image d'un je qui ne raconte pas seulement des faits (narrating self) mais les a vécus (experiencing self).

23. Froissart, Extrait 3, page 611, lignes 28-30

Et je, auteur de ce livre, qui fus présent à toutes ces choses, quand j'en vis si grand'foison, je me merveillai où l'on en avoit tant pris ;

D'autre part, lorsque le je est présenté comme un témoin indirect, c'est-à-dire qu'il a lu ou entendu raconter un événement, il peut être confondu avec le narrateur-auteur en tant qu'être du monde qui a rassemblé des sources pour rédiger son œuvre (narrating self). ${ }^{15}$ 


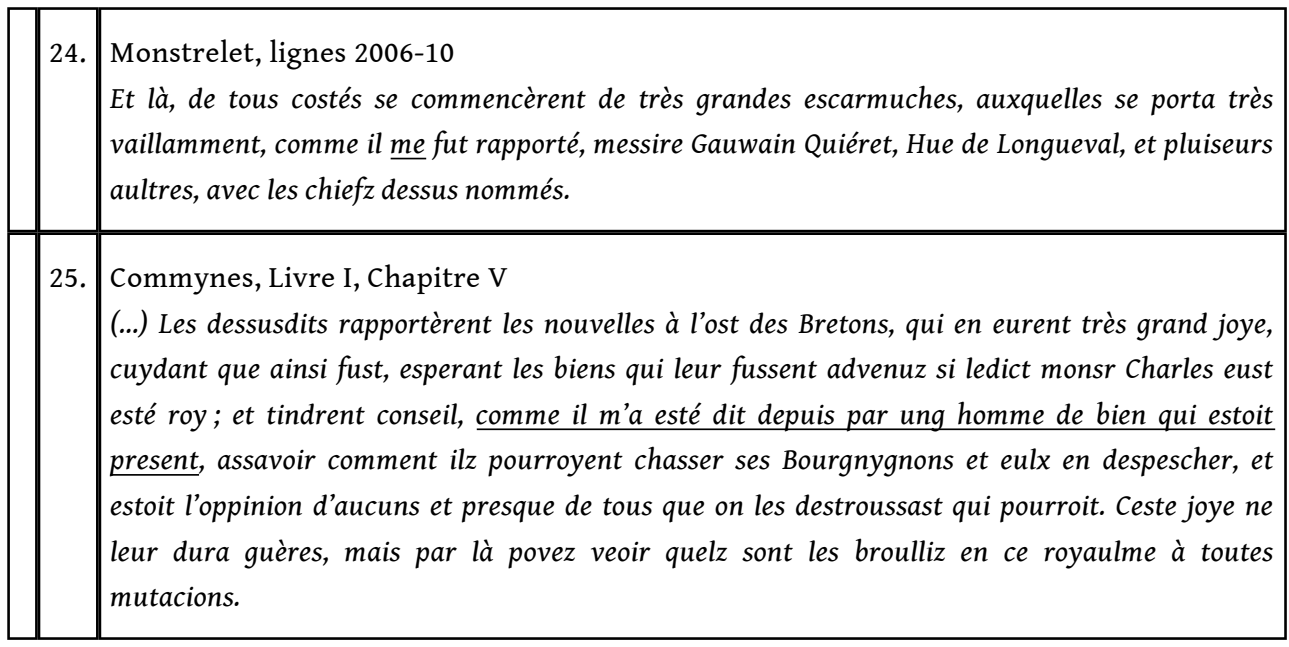

Comme je l'ai déjà noté, certaines chroniques comme celle de Joinville, Froissart 2,3 et Commynes ne réfèrent pas seulement au je en tant que témoin direct et indirect mais aussi en tant que participant aux événements racontés ${ }^{16}$. Les trois extraits de Froissart sont particulièrement intéressants car ils présentent des facettes différentes. L'extrait 1 est tiré du premier livre dans lequel Froissart raconte des événements auxquels il n'a pas assisté, en se basant notamment sur le texte de Jean le Bel; il se décrit dès lors principalement en tant que narrateur-auteur. Dans l'extrait 3, tiré du livre IV, Froissart se présente en tant que narrateur-auteur (76\%) mais aussi en tant que témoin direct et indirect (17\%). Toutefois il n'insiste pas beaucoup sur ses propres activités au moment des faits racontés (5\%). L'extrait 2 de Froissart offre des caractéristiques différentes des extrait 1 et 3 et des autres chroniques ${ }^{17}$. Dans le Voyage de Bearn, Froissart raconte son voyage par étapes pour atteindre la cour de Gaston Phébus et son séjour à la cour de ce dernier, période durant laquelle il a l'occasion de passer de longs moments avec des informateurs qui lui racontent des événements importants auxquels ils ont assisté et participé, et lui donnent des renseignement sur les régions qu'il visite en leur compagnie ainsi que sur la vie même de Gaston Phébus. Dans cet extrait, Froissart se représente à de nombreuses reprises en tant que personnage/voyageur et "reporter" ainsi qu'en tant qu'écrivain-chroniqueur puisqu'il indique à ses informateurs qu'il notera leurs témoignages dans ses chroniques et qu'il précise dans son récit comment il écrit toutes ces histoires lorsqu'il se retrouve dans sa chambre d'hôtel en fin de journée.

Froissart 2 emploie un nombre élevé de $1^{\text {ères }}$ pers. du sg. pour se désigner en tant que narrateur d'une part (2.7\%) et en tant que personnage-témoin d'autre part (37.6\%) ${ }^{18}$. Il utilise aussi un très grand nombre de discours directs ( $80 \%$ de l'espace du récit), dont plusieurs ont été prononcés par lui-même au sein de dialogues avec les informateurs. La fonction du discours direct est ici certainement moins de dramatisatiser un événement historique mais plutôt de mettre en scène et donc d'authentifier la récolte des sources ${ }^{19}$. Il semble donc que la mise en scène du je et des discours directs n'apparaissent pas pour impliquer les auditeurs-lecteurs dans la narration (absence relative du vous) mais plutôt pour insister sur la vraisemblance, la vérisimilitude du processus de témoignage indirect : le Voyage présente Froissart «reporter » interrogeant un témoin direct des événements ainsi que Froissart écrivain rédigeant ses notes et parlant de son œuvre. Par là-même, l'extrait constitue une représentation de son travail d'historien, une «chronique des 
Chroniques» (Diller 1998: 57) qui vient garantir la vérité de l'ensemble du travail, au même titre que les allusions à la fonction testimoniale directe dans les textes de Joinville ( $1.3 \%$ de $1^{\text {ères }}$ pers. du sg.) et de Commynes ( $3.5 \%$ de $1^{\text {ères }}$ pers. du sg.) et que leurs rôles de participants à l'action ( $21 \%$ de $1^{\text {ères }}$ pers. du sg. chez Joinville et $1.3 \%$ dans l'extrait de Commynes). Cette mise-en-scène originale est essentiellement restreinte au Voyage de Bearn, puisqu'il y a un retour à la narration plus traditionnelle dans le reste du livre III et dans le livre IV (extrait 3). Cependant elle renforce plutôt qu'elle ne remet en cause les observations faites pour le livre I et les autres textes. Il y a en effet dans les chroniques une volonté délibérée de souligner le travail de création et d'écriture des chroniqueurs en tant que narrateurs-auteurs ainsi que leur position de témoins et parfois personnages, garants de la vérité des événements racontés. Comme le décrit si bien Michèle Perret (1981: 2-3), dès le texte de Joinville, «l'engagement personnel du témoin » et «l'inscription du narrateur dans son récit » se font caution de la vérité de la narration.

\section{BIBLIOGRAPHIE}

AINSWORTH, Peter F. (1998) « Configuring Transience : Patterns of Transmission and Transmissibility in the Chroniques (1395-1995) ». Dans Maddox, Donald et Sara Sturm-Maddox (éds.). Froissart across the Genres. Gainesville, Fl. : University Press of Florida. 15-39.

AINSWORTH, Peter F. (1990) Jean Froissart and the Fabric of History: Truth, Myth and Fiction in the Chroniques. Oxford : Clarendon.

BAUMGARTNER, Emmanuèle (1995) Le Récit médiéval. Paris : Hachette.

BLANCHARD, Joël (1991) «Commynes et l'historiographie de son temps ». Recherches sur la littérature du XVe siècle. Actes du Vie Colloque international sur le Moyen Français. Milan : Vitae Pensiero Publicazioni dell’ Università Cattolica. 191-205.

CALIN, William (1993) « Narrative Technique in Fourteenth-Century France : Froissart and his Chroniques ». Dans Rupert T. Pickens (éd.). Studies in Honor of Hans-Erich Keller. Kalamazoo, Michigan : Medieval Institute Publications Western Michigan University. 227-36.

DILLER, G. T. (1998) « Froissart's 1389 Travel to Béarn : A Voyage Narration to the Center of the Chroniques ». Dans Maddox, Donald et Sara Sturm-Maddox (éds.). Froissart across the Genres. Gainesville, Fl. : University press of Florida. 50-60.

DUFOURNET, Jean (1994) Philippe de Commynes : un historien à l'aube des temps modernes. Bruxelles : De Boeck Université.

DUFOURNET, Jean (1982) Sur Philippe de Commynes : quatre études. Paris : Société d'édition d'enseignement supérieur.

DUFOURNET, Jean (1969) « Art et déformation historique dans les Mémoires de Philippe de Commynes ». Romania. 90, $358: 2.146-73$.

FLEISCHMAN, Suzanne (1983) « On the Representation of History and Fiction in the Middle Ages ». History and Theory, Studies in the Philosophy of History, 23. 3. 278-310. 
JACQUIN, Gérard (1993) « Robert de Clari, témoin et conteur ». Dans Et c'est la fin pour quoy nous sommes ensemble. Hommage à Jean Dufournet; Littérature, Histoire et Langue du Moyen Age. Tome 2. Paris : Champion, 747-757.

MARCHELLO-NIZIA, Christiane (1984) « L'Historien et son prologue ». Dans Poirion, Daniel (éd.). La Chronique et l'histoire au Moyen-Âge. Paris : Cultures et civilisations médiévales. 2. 13-25.

MARNETTE, Sophie (2002) « Sources du récit et discours rapportés : L'art de la représentation dans les chroniques et les romans français des $14^{\mathrm{e}}$ et $15^{\mathrm{e}}$ siècles ». À paraître dans La Revue du Moyen Français. Actes du Xe Colloque international sur le moyen français.

MARNETTE, Sophie (2001) «Aux frontières du discours rapporté ». À paraître dans Revue Romane. 36. 2.

MARNETTE, Sophie (1999) « Narrateur et points de vue dans les chroniques médiévales : une approche linguistique ». Dans Erik Kooper (éd.). The Medieval Chronicle, Proceedings of the $1^{\text {st }}$ International Conference on the Medieval Chronicle, Utrecht 13-16 July 1996. Amsterdam / Atlanta : Rodopi. 174-190.

MARNETTE, Sophie (1998) Narrateur et points de vue dans la littérature française médiévale : Une approche linguistique. Bernes : Peter Lang.

PALMER, J.J.N (éd.) (1981) Froissart : Historian. Woodbridge : Boydell.

PERRET, Michèle (1997) « Le discours rapporté dans Le bel inconnu ». L'Information grammaticale, 72. 13-18.

PERRET, Michèle (1995) « Histoire, nomination, référence ». dans Michèle Perret (éd.). Linguistique de l'énonciation. Approche diachronique. Linx, 32. 173-191.

PERRET, Michèle (1993) « Writing History / Writing Fiction ». Actes du Colloque inernational : Mélusine at 600, Amherst (Massachussett), 5-7 Novembre 1993.

PERRET, Michèle (1988) Le Signe et la mention. Adverbes embrayeurs ci, ça, la, illuec en moyen français (XIV ${ }^{e}-X V^{e}$ siècles). Genève : Droz.

PERRET, Michèle (1981) « A la fin de sa vie ne fuz-je mie... ». Revue des sciences humaines, 55. 183. $17-37$

SOUTET, Olivier \& Claude THOMASSET (1984) «Des Marques de la subjectivité dans les Mémoires de Commyne ». Dans Poirion, Daniel (éd.). La Chronique et l'histoire au Moyen-Âge. Paris : Cultures et civilisations médiévales. 2.27-44.

SPIEGEL, Gabrielle M. (1993) Romancing the Past. The Rise of Vernacular Prose Historiography in Thirteenth-Century France. Berkeley and Los Angeles, California : University of California Press.

ZINK, Michel (1990) Littérature française : le moyen âge. Nancy : Presses Universitaires de Nancy.

ZINK, Michel (1985) La Subjectivité littéraire autour du siècle de Saint Louis. Paris : Presses Universitaires de France.

\section{NOTES}

1. Ici, j'entendrai par "auteur", ce qui est posé par le texte comme l'instance créatrice, à l'origine du récit tandis que le «narrateur » est l'instance qui raconte. Les difficultés posées par ces notions en littérature médiévale et la différence entre écrivain (personne réelle) et auteur (création textuelle) sont développées dans un ouvrage précédent (voir Marnette 1998 :18-19 et 
216-20). Dans les récits à tendance impersonnelle, le je est pratiquement absent du texte mais latent car l'instance narrative peut à certaines reprises émettre des opinions et présenter les événements selon une perspective qui est extérieure à celles des personnages (voir Marnette 1998 : 17-22).

2. Corpus par ordre chronologique :

Villehardouin, La Conquête de Constantinople, composé avant 1212, Extrait correspondant au premier tome de l'édition de Faral, Paris : Les Belles lettres, 1961.

Robert de Clari, La Conquête de Constantinople, composé après 1216 , édité par Lauer, Paris: Champion, 1924.

Jehan, Seigneur de Joinville. La Vie de Saint Louis. Le témoignage de Jehan, Seigneur de Joinville, composé en 1309. Edité par Noel Corbett, Québec : Naaman, 1977.

Froissart, Chroniques, Extrait 1: Manuscrit d'Amiens, version composée vers 1380, éditée par George T. Diller, Genève : Droz, 1991, 5 volumes. Extrait tiré du volume 1, §§ 1-100, p. 1-131.

Froissart, Chroniques, Extrait 2 : 'Le voyage de Bearn' Manuscrit de Rome, version composée vers 1400, paru dans l'anthologie d'A. Pauphilet et E. Pognon, Historiens et Chroniqueurs du moyen âge, Paris : Gallimard, 1952. Extrait tiré du volume 3 des chroniques, §§ 1-6, p. 486-565.

Froissart, Chroniques, Extrait 3 : Manuscrit de Rome, version composée vers 1400, paru dans l'anthologie d'A. Pauphilet et E. Pognon, Historiens et Chroniqueurs du moyen âge, Paris : Gallimard, 1952. Extrait tiré du volume 4 des chroniques, §§ 5-18, p. 605-647.

Enguerran de Monstrelet, Chronique, composé entre 1444 et 1453, 2 livres, édité par L. DouëtD’Arq, Société de l'histoire de France, 1857. Extrait tiré du Livre II, chapitres 258-278.

Gilles Le Bouvier dit Le Héraut Berry, Les Chroniques du roi Charles VII, composé vers 1455, édité par H. Courteault et L. Celier, Paris : Klincksieck, 1979. p. 3-4, p. 122-99.

Commynes, Mémoires, composé vers 1490, édité par J. Calmette, Paris : Belles Lettres, 1964-5, 8 volumes. Extrait tiré du volume 1 (16 chapitres).

L'analyse des textes en moyen français a été en grande partie rendue possible par l'accès au corpus et au moteur de recherche de l'équipe 'Linguistique et Informatique' de l'ENS de Fontenay-Saint-Cloud, dirigée par Christiane Marchello-Nizia, que je remercie infiniment.

3. Voir aussi , entre autres, Zink (1990, chap. 6), Baumgartner (1995, chap. 4).

4. Si l'on compte la fréquence d'emploi des premières et deuxièmes personnes (pronoms personnels, adjectifs possessifs et personnes verbales) dans les chroniques de Clari et Villehardouin, le taux de $1^{\text {ères }}$ pers. du sg. est inférieur à $1 \%$ et moins élevé que le taux de $1^{\text {ères }}$ pers. du pl. (1.5\% et $2 \%)$ et de $2^{\text {es }}$ pers. (1.3\% et $\left.3.2 \%\right)$.

5. Bien sûr, comme Fleischman (1983: 293) le fait très bien remarquer, la sélection même des événements racontés est une preuve de la subjectivité du récit et donne à celui-ci une signification particulière. Zink (1985 : 209-212) souligne lui-aussi la subjectivité des textes de Villehardouin et de Clari. En effet, malgré un ton apparemment impersonnel, ces deux textes transmettent un certain message : Villehardouin a à cœur de justifier la décision des Croisés de passer par Venise et puis d'aller conquérir Constantinople, décision à laquelle il a grandement participé, étant l'un des négociateurs du traité passé avec Venise. Clari quant à lui apporte une vision différente, l'expérience d'un chevalier pauvre qui n'a pas participé aux décisions des 'grands' mais qui a vécu les épreuves des Croisés; il porte rancune à certains seigneurs et exprime son admiration pour d'autres (voir aussi Jacquin 1993).

6. Calcul : Si l'on examine le nombre de références à la $1^{\text {ère }}$ pers. sg. dans un texte, on obtient un nombre $\mathrm{x}$ de $1^{\text {ères }}$ pers. sg. pour un nombre y de lignes, selon le texte envisagé, sachant que l'on peut avoir plusieurs occurrences par ligne. En divisant le nombre total de $1^{\text {ères }}$ pers. sg. (x) par le nombre total de lignes du texte (y) de la partie narrative et en le multipliant par 100, on obtient un pourcentage. Comme les éditions des textes envisagés présentent des lignes de longueur différente, il faut aussi transformer le nombre de lignes total des textes en un nombre de syllabes puis le diviser par 10 pour le faire correspondre à un système décasyllabique. Application : 80 
occurrences de $1^{\text {ères }}$ pers. sg. dans Froissart 1 pour 2305.5 lignes dans la partie narrative. Il y a environ 18 syllabes par ligne. $80 \div((2305.5 \times 18) \div 10) \times 100=1.9 \%$. Le nombre décimal de lignes (2305.5) s'explique par le fait que j'ai compté le nombre de lignes 'entières', c'est-à-dire en déduisant du nombre total de lignes les espaces blancs de début ou de fin de paragraphe ou suivant les discours directs.

7. Sur l'importance du je et de la fonction testimoniale dans les chroniques des $14^{\mathrm{e}}$ et $15^{\mathrm{e}} \mathrm{s}$., voir Perret (1981) pour Joinville ; Blanchard (1991) et Soutet \& Thomasset (1984) pour Commynes; ainsi que Ainsworth (1990, 1998), Calin (1993), Diller (1998), Dufournet $(1969,1982,1994)$ et Palmer (1981) pour Froissart.

8. Les fréquences globales d'emploi de la 1 ère pers. du sg. sont de $0.7 \%$ pour Monstrelet et de $1.5 \%$ pour Berry mais elles tombent à $0.2 \%$ si on considère uniquement le corps des textes, sans les prologues où le chroniqueur présente son œuvre.

9. Le nom de Commynes n'apparaît pas dans l'extrait choisi pour mon corpus mais il est présent dans d'autres parties de son œuvre.

10. Dans le Tristan en prose et d'autres romans cités par Marchello-Nizia (1984:21), l'expression à la $1^{\text {ère }}$ pers. qui suit le " je+ nom propre " a trait à l'activité de traduction et non à celle de témoignage.

11. En bref, la différence entre narrateur en tant que tel et narrateur en tant qu'être du monde se base sur la distinction que Ducrot (1984:199) effectue à l'intérieur même de la notion de locuteur entre : «(...) le locuteur en tant que tel (par abréviation "L") et le locuteur en tant qu'être du monde (“ $\lambda$ ”). L est le responsable de l'énonciation considéré uniquement en tant qu'il a cette propriété, $\lambda$ 區est une personne "complète », qui possède, entre autres propriétés, celle d'être à l'origine de l'énoncé (...) ».

Ainsi, l'énoncé «je suis quelqu'un de franc » attribue-t-il au locuteur- $\lambda[$ [0:[2: certaine qualité indépendante de l'énonciation. En revanche, dans l'énoncé «franchement, il exagère ", l'adverbe "franchement » est lié au locuteur-L responsable de l'énonciation mais pas au locuteur- $\lambda$ 血 Dans Je vous dis que $X$, le pronom « je » désigne à la fois le locuteur- $\mathrm{L}$, le locuteur- $\lambda$ et l'énonciateur. En bref, l'énoncé Je vous dis que X exprime deux choses différentes : un acte de langage et la mise en scène de cet acte de langage. Pour une discussion approfondie de ce type de discours rapporté, voir Marnette (2001).

12. Si une expression comme je dis que exprime un acte de langage au sens d'acte de "parole ", les expressions comme je pense (que), je crois (que), que certains nomment « rapport d'actes de pensées "ne fonctionnent pas forcément de façon entièrement parallèle. Ainsi on ne réalise pas l'action de penser en disant « je pense que c'est vrai », ni l'action de croire en disant " je crois que c'est vrai » car, contrairement à l'acte de « parole », l'acte de pensée ne peut être accompli par le langage, il peut seulement être décrit par le biais de celui-ci. Cependant le point commun de ces deux types d'expressions est qu'elles contiennent un verbe au présent et à la première personne et qu'il s'agit donc de ce que j'appelle des discours rapportés « réflexifs » (Marnette, 2001).

13. Voir Marnette (2002) pour les chiffres détaillés et une comparaison avec les romans en prose. 14. Ajoutons cependant que l'impossibilité certaine de rapporter les paroles telles qu'elles ont vraiment été prononcées (ou même les pensées) n'empêche pas en principe l'emploi du discours direct. En effet, le problème est exactement le même dans les récits naturels en français parlé mais le discours direct y est au moins aussi souvent utilisé que le discours indirect pour rapporter des paroles, ce qui permet au locuteur citant de donner vie au récit qu'il raconte et d'y impliquer ses allocutaires (Marnette 2001). La préférence des chroniques pour le discours indirect maintient au contraire les discours dans le passé durant lequel ils se sont produits et à distance des auditeurslecteurs : il s'agit donc d'une vérité historique objectivisée, mise à distance. Par opposition aux chansons de geste où les événements historiques sont re-vécus au fur et à mesure qu'ils sont représentés par la voix du narrateur-jongleur (Marnette 1998 : 135, 1999 : 177-8, 185).

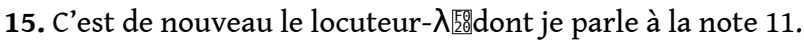


16. Joinville ( $\mathrm{e}^{\mathrm{e}}$ pers sg. $21 \%, 1^{\mathrm{e}}$ pers pl. $\left.12.5 \%\right)$, Froissart $2(37.6 \%, 13.5 \%)$, Froissart $3(5 \%)$, Commynes $(1.3 \%, 2.7 \%)$.

17. On peut se référer aux excellents travaux d'Ainsworth (1990:140-71, 1998 :17-21), Calin (1993) et Diller (1998) pour des analyses détaillées.

18. S'ajoute à cela $13.5 \%$ de $1{ }^{\text {ères }}$ pers. pl.

19. Comme le fait remarquer Ainsworth (1990: 150-1), les voix des informateurs de Froissart ressemblent fort à la sienne propre, avec les mêmes tics de langage et des expressions similaires. Diller (1998 : 57) fait même l'hypothèse que l'un des informateurs, Espan de Lyon, est en fait une création fictive de Froissart, ce qui renforce encore notre impression qu'il y a mise-en-scène.

\section{AUTEUR}

\section{SOPHIE MARNETTE}

Zaharoff Fellow in French Linguistics -

University of Oxford - Oxford OX12JF

Royaume Uni 